\title{
FGFR3-targeted mAb therapy for bladder cancer and multiple myeloma
}

\author{
Yaron Hadari' and Joseph Schlessinger ${ }^{2}$ \\ ${ }^{1}$ Kolltan Pharmaceuticals Inc., New Haven, Connecticut, USA. 'Department of Pharmacology, \\ Yale University School of Medicine, New Haven, Connecticut, USA.
}

\begin{abstract}
Gain-of-function mutations in FGF receptor 3 (FGFR3) have been implicated in severe skeletal dysplasias and in a variety of cancers. In their study in this issue of the JCI, Qing et al. used specific shRNA probes to demonstrate that FGFR3 functions as an important driver of bladder carcinoma cell proliferation (see the related article beginning on page 1216). A unique anti-FGFR3 $\mathrm{mAb}$ was shown to exhibit antitumor activity in human bladder carcinoma cells in vitro and in mouse bladder cancer or multiple myeloma xenograft tumor models bearing either wild-type or mutant FGFR3. These results suggest that clinical development of anti-FGFR $3 \mathrm{mAbs}$ should be considered for targeted therapy of cancer and other diseases.
\end{abstract}

FGFs are members of a large family of growth factors that play an important role in the control of diverse cellular processes (e.g., cell proliferation, differentiation, survival, and migration) during embryonic development and in the maintenance of cellular homeostasis in virtually all organs and tissues $(1,2)$. FGFs act in concert with heparan sulfate proteoglycans (HSPGs) to activate a family of four receptor tyrosine kinases (RTKs) designated FGF receptors 1-4 (FGFR1-4). Each FGFR consists of an extracellular region composed of three Iglike domains designated D1, D2, and D3; a short stretch of amino acids in the D1-D2 linker region, designated the acid box; and a single transmembrane domain followed by an intracellular tyrosine kinase domain with additional regulatory sequences (1). Diversity in ligand-binding specificity and tissue expression patterns of FGFR1-3 are further enhanced by alternative RNA splicing in the $\mathrm{C}$-terminal half of $\mathrm{D} 3$ to generate either the IIIb or the IIIc splice isoform. It was previously shown that the IIIb isoform is expressed in epithelial cells and the IIIc isoform is expressed in mesenchymal cells (1). Interestingly, FGFs that activate the epi-

Conflict of interest: $Y$. Hadari is an employee of and owns equity in Kolltan Pharmaceuticals Inc. J. Schlessinger owns equity in and has received consultancy fees from Plexxikon Inc. and Kolltan Pharmaceuticals Inc., which develop drugs for the treatment of cancer and other diseases.

Nonstandard abbreviations used: FGFR, FGF receptor; RTK, receptor tyrosine kinase.

Citation for this article: J. Clin. Invest. 119:1077-1079 (2009). doi:10.1172/JCI38948. thelial IIIb FGFR isoforms are expressed in mesenchymal cells, whereas FGFs that activate the mesenchymal IIIc FGFR isoforms are produced in epithelial cells (1).

A variety of human skeletal dysplasias associated with severe impairment in cranial, digital, and skeletal development have been shown to be driven by gain-of-function mutations in FGFR1-3 (1). Gain-of-function mutations in FGFRs have also been identified in a variety of human cancers, including myeloproliferative syndromes, lymphomas, glioblastoma, as well as prostate, bladder, and mammary carcinomas (1). Importantly, the $\mathrm{t}(4 ; 14)(\mathrm{p} 16.3 ; \mathrm{q} 32)$ chromosomal translocation that results in FGFR3 overexpression in $20 \%$ of multiple myeloma patients is correlated with poor clinical response and patient survival (3). FGFR3 overexpression has also been identified in bladder carcinoma (4). Moreover, somatic gain-of-function mutations in FGFR3 have been identified in $65 \%$ of papillary and in $20 \%$ of muscle-invasive bladder carcinomas (4). These studies suggest that FGFR3 could be considered a candidate for targeted therapies for the treatment of cancers and skeletal dysplasias drivSeveral small molecule inhibitors of the tyrosine kinase activity of FGFR3 and other members of the FGFRs have been described, some of which are currently in clinical development $(5,6)$. However, potent small molecule inhibitors that specifically interfere with the tyrosine kinase activity of FGFR3 are difficult to develop because of the close structural similarity of the FGFR3 tyrosine kinase domain to that of other members of en by gain-of-function FGFR3 mutations. the FGFR family and other RTKs. An alternative approach for developing a selective inhibitor of FGFR 3 that does not interfere with the activity of other FGFRs and RTKs is to raise inhibitory mAbs that selectively bind to the extracellular domain of FGFR3.

\section{Development of a specific anti- FGFR3 mAb}

Since the therapeutic potential of targeting FGFR3 for the treatment of bladder cancer has not been well defined, in their study in this issue of the JCI Qing et al. first examined the role played by activating FGFR3 mutations in bladder cancer by employing specific shRNA probes that silence FGFR3 expression in the tumor cells (7). Using several human bladder cancer cell lines expressing either wild-type or activated FGFR3 mutants, Qing et al. demonstrated that FGFR3 plays a major role in bladder cancer cell proliferation and that silencing of FGFR3 expression leads to attenuation of cell-cycle progression rather than stimulation of cell apoptosis.

Qing et al. went on to generate an antiFGFR3 $\mathrm{mAb}$, via the immunization of mice and by screening phage display libraries for $\mathrm{mAbs}$ that bind to the extracellular region of FGFR 3 and thereby interfere with FGF binding (7). The most effective antagonist of FGFR3 activity identified in these experiments was an anti-FGFR $3 \mathrm{mAb}$ the authors termed R3Mab. The authors demonstrate that R3Mab binds selectively to FGFR 3 and interferes with ligand binding to either the IIIb or IIIc isoforms of FGFR3. Moreover, R3Mab blocked FGF1-mediated stimulation of FGFR3 activation, tyrosine phosphorylation of the docking protein FGFR substrate $2 \alpha$, the MAPK response, and FGFR3-dependent proliferation of normal and transformed cells (Figure 1).

Detailed studies of R3Mab binding to a variety of synthetic peptides derived from the extracellular region of FGFR3, as well as the determination of the X-ray crystal structure of $\mathrm{R} 3 \mathrm{Mab}$ in complex with the $\mathrm{D} 2$ and D3 domains of FGFR3, revealed that 


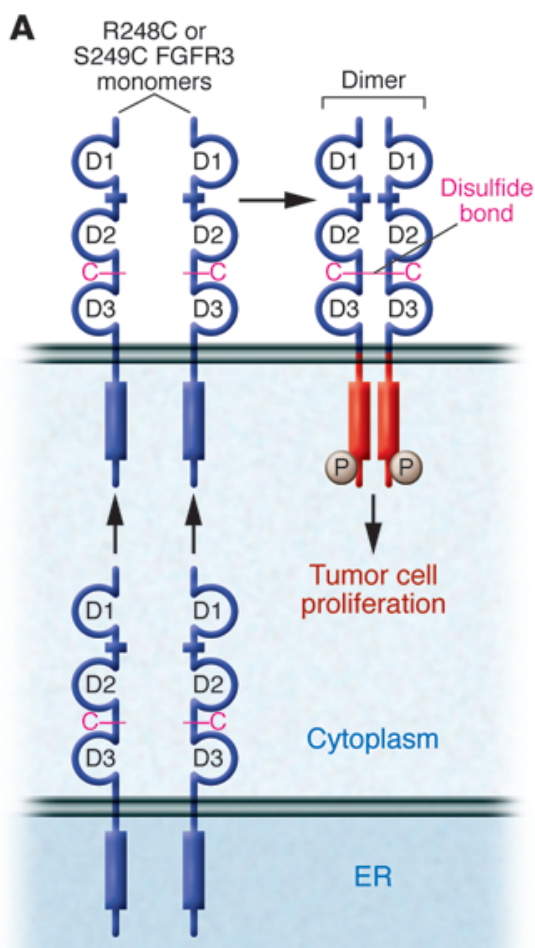

R3Mab binds to epitopes corresponding to FGF- and HSPG-binding sites in D2 and D3 and to epitopes in a region responsible for mediating receptor-receptor contacts between neighboring dimeric FGFR3 molecules $(7,8)$. The X-ray crystal structure also showed that R3Mab binds preferentially to an alternate conformation of $\mathrm{D} 3$ relative to $\mathrm{D} 2$, one that is different from the orientation of D3 relative to D2 that was seen in the crystal structure of FGF1 in complex with D2 and D3 of FGFR3 (7-9).

Most FGFR3-activating mutations identified in bladder cancer are located in the extracellular domain of the receptor (4). These mutations (R248C or S249C) give rise to a new, unpaired cysteine residue, leading to formation of disulfide-linked FGFR3 dimers in a ligand-independent manner. The disulfide-linked FGFR3 dimers exhibit constitutive tyrosine kinase activity and ligand-independent stimulation of cellular signaling pathways (4) (Figure 1). Qing et al. show that, remarkably, R3Mab exhibits a robust inhibitory activity not only against wild-type FGFR3 but also against a variety of oncogenic FGFR3 mutants, including the oncogenic, disulfide-linked R248C or S249C FGFR3 mutants (7) (Figure 1). Interestingly, it is demonstrated that formation of disulfide-linked FGFR3
B

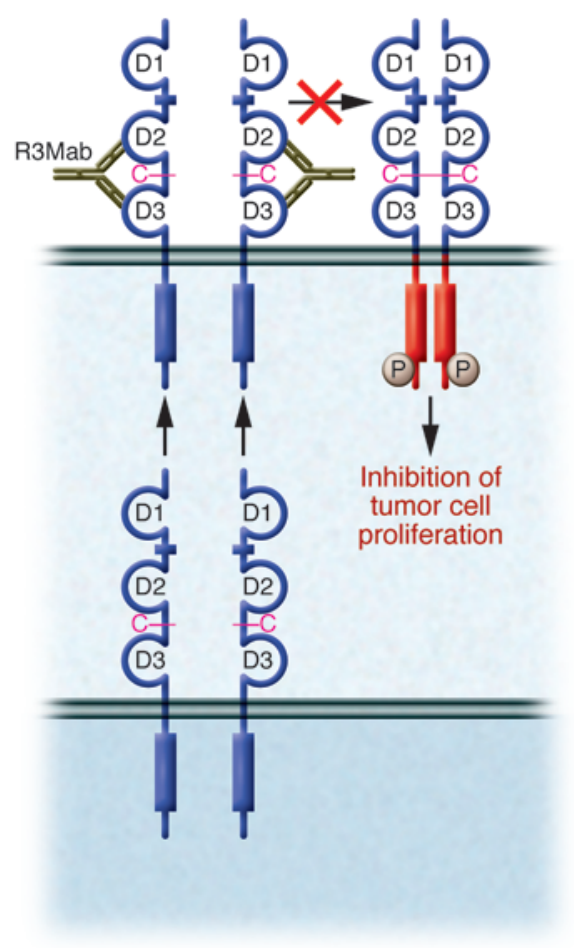

Figure 1

Potential mechanism of inhibition of the activity of disulfide-linked oncogenic FGFR3 mutants by treatment with an anti-FGFR3 mAb (R3Mab). Oncogenic FGFR3 mutants $\mathrm{R} 248 \mathrm{C}$ and S249C remain monomeric as long as they remain inside the cell in the endoplasmic reticulum and other intracellular compartments. Upon migration to the cell surface and exposure to the oxidative extracellular environment, disulfide-linked activated dimers of FGFR3 are formed, resulting in tyrosine phosphorylation and triggering of intracellular signaling pathways that result in bladder cell carcinoma proliferation (A). In their study in this issue of the $\mathrm{JCl}$, Qing et al. (7) report that binding of their anti-FGFR3 mAb (R3Mab) to D2 and D3 of the extracellular ligand-binding region of FGFR3 before intermolecular disulfide linkages are formed prevents formation of disulfide-linked FGFR3 dimers with constitutively stimulated tyrosine kinase activity, resulting in the inhibition of bladder cell carcinoma proliferation and of tumor formation (B). Cysteines of R248C or S249C FGFR3 are shown in magenta. The stimulated tyrosine kinase domain is shown in red. dimers is prevented by $\mathrm{R} 3 \mathrm{Mab}$ treatment or by treating the cells with a non-cell-permeating agent that blocks free sulfhydryl groups from forming disulfide linkages. Qing et al. propose that a reversible equilibrium between the monomeric and disulfide-linked oncogenic forms of FGFR3 exists and that R3Mab binding shifts the equilibrium toward the monomeric inactive state, resulting in inhibition of FGFR3 activity. Since disulfide bond formation is not a reversible chemical reaction, an alternative and more plausible mechanism is that the R248C or S249C FGFR3 mutants remain monomeric as long as they are located inside the cell due to the reducing intracellular environment. After the R248C or S249C FGFR3 mutants are transported to the cell surface, the unpaired cysteine of the (R248C or S249C) FGFR3 mutant pairs with the unpaired cysteine of another FGFR3 mutant molecule to generate a disulfide-linked FGFR3 dimer - a process likely facilitated by the oxidative nature of the extracellular environment. As mutant FGFR3 is transported to the cell surface in a monomeric configuration, the rate of disulfide bond formation between neighboring mutant monomers must be sufficiently slow to permit R3Mab binding and prevention of disulfide bond forma- tion between the R248C or S249C FGFR3 mutants, even in the oxidative extracellular environment (Figure 1).

\section{Efficacy of R3Mab therapy in murine tumor models}

Qing et al. show that R3Mab treatment of bladder cancer or multiple myeloma xenografts in mice has an antitumor effect on tumors bearing either wild-type or mutant FGFR3 (7). These experiments demonstrate that $\mathrm{R} 3 \mathrm{Mab}$ is a potent inhibitor of FGFR3-driven tumors in vivo and suggest that R3Mab is a good candidate for clinical development. However, whereas accumulation of mutant FGFR3 monomers was detected in lysates from cultured human bladder cancer cells treated with R3Mab, Qing et al. were not able to detect a similar accumulation of mutant FGFR3 monomers in lysates from tumors isolated from mice treated with R3Mab. This difference may be due to technical reasons; alternatively, the antitumor activities of R3Mab in vitro and in vivo may be mediated by different mechanisms. An intriguing possibility is that treatment of the tumor-bearing mice with antioxidants may potentiate the in vivo antitumor activity of R3Mab.

As noted above, robust antitumor activity of R3Mab was also detected in mice 
bearing multiple myeloma xenografts (7). The multiple myeloma tumors that were treated with R3Mab overexpress wild-type FGFR3. It was convincingly demonstrated that the antitumor effect of R3Mab treatment of tumor cells bearing high amounts of wild-type FGFR3 on their cell surface was mediated in part by Ab-dependent cell-mediated cytotoxicity (ADCC). By contrast, ADCC did not seem to play a role in the antitumor effect of R3Mab on human bladder cancer cells, which express at least a 5-fold lower level of wild-type or mutant FGFR 3 compared with the multiple myeloma cells studied.

Finally, since similar gain-of-function mutations in FGFR3 cause skeletal dysplasias and other developmental disorders, it will be important in future studies to examine whether R3Mab treatment may prevent or attenuate developmental disorders caused by FGFR3-activating mutations in neonatal murine models of these diseases (10-12).

\section{Acknowledgments}

This work was supported by NIH grants R01-AR051448, R01-AR051886, and P50AR054086 to Joseph Schlessinger.

Address correspondence to: Joseph Schlessinger, Department of Pharmacology, Sterling Hall of Medicine, B-204, 333 Cedar Street, Yale University School of Medicine, New Haven, Connecticut 06520-8066, USA. Phone: (203) 7857395; Fax: (203) 785-3879; E-mail: joseph. schlessinger@yale.edu.

1. Eswarakumar, V.P., Lax, I., and Schlessinger, J. 2005. Cellular signaling by fibroblast growth factor receptors. Cytokine Growth Factor Rev. 16:139-149.

2. Dailey, L., Ambrosetti, D., Mansukhani, A., and Basilico, C. 2005. Mechanisms underlying differential responses to FGF signaling. Cytokine Growth Factor Rev. 16:233-247.

3. Chesi, M., et al. 1997. Frequent translocation $\mathrm{t}(4 ; 14)(\mathrm{p} 16.3 ; \mathrm{q} 32.3)$ in multiple myeloma is associated with increased expression and activating mutations of fibroblast growth factor receptor 3 . Nat. Genet. 16:260-264.

4. Knowles, M.A. 2008. Novel therapeutic targets in bladder cancer: mutation and expression of FGF receptors. Future Oncol. 4:71-83.

5. Trudel, S., et al. 2004. CHIR-258, a novel, multitargeted tyrosine kinase inhibitor for the potential treatment of $\mathrm{t}(4 ; 14)$ multiple myeloma. Blood. 105:2941-2948.

6. Grand, E.K., Chase, A.J., Heath, C., Rahemtulla, A., and Cross, N.C. 2004. Targeting FGFR3 in multiple myeloma: inhibition of $\mathrm{t}(4 ; 14)$-positive cells by SU5402 and PD173074. Lenkemia. 18:962-966.

7. Qing, J., et al. 2009. Antibody-based targeting of FGFR3 in bladder carcinoma and $\mathrm{t}(4 ; 14)$-positive multiple myeloma in mice. J. Clin. Invest. 119:1216-1229.

8. Plotnikov, A.N., Schlessinger, J., Hubbard, S.R., and Mohammadi, M. 1999. Structural basis for FGF receptor dimerization and activation. Cell. 98:641-650.

9. Schlessinger, J., et al. 2000. Crystal structure of a ternary FGF-FGFR-heparin complex reveals a dual role for heparin in FGFR binding and dimerization. Mol. Cell. 6:743-750.

10. Chen, L., et al. 1999. Gly369Cys mutation in mouse FGFR3 causes achondroplasia by affecting both chondrogenesis and osteogenesis. J. Clin. Invest. 104:1517-1525.

11. Twigg, S.R., et al. 2009. Skeletal analysis of the Fgfr3(P244R) mouse, a genetic model for the Muenke craniosynostosis syndrome. Dev. Dyn. 238:331-342.

12. Pannier, S., et al. 2009. Activating Fgfr3 Y367C mutation causes hearing loss and inner ear defect in a mouse model of chondrodysplasia. Biochim. Biophys. Acta. 2:140-147.

\title{
Anti-TNF immunotherapy and tuberculosis reactivation: another mechanism revealed
}

\author{
Elizabeth A. Miller1,2 and Joel D. Ernst'1,3,4
}

${ }^{1}$ Division of Infectious Diseases, Department of Medicine, ${ }^{2}$ Cancer Institute, ${ }^{3}$ Department of Pathology, and ${ }^{4}$ Department of Microbiology, New York University School of Medicine, New York, New York, USA.

\begin{abstract}
Anti-TNF immunotherapy has revolutionized the treatment of some inflammatory diseases, such as RA. However, a major concern is that patients receiving this therapy have an increased risk of fungal and bacterial infection, particularly of reactivating latent tuberculosis (TB). In this issue of the JCI, in an effort to understand how anti-TNF immunotherapy affects host mechanisms required to control TB, Bruns and colleagues examined the effects of the anti-TNF therapeutic infliximab on Mycobacterium tuberculosis-specific human lymphocytes (see the related article beginning on page 1167). The authors report that a granulysinexpressing $\mathrm{CD}^{2} \mathrm{RA}^{+}$subset of effector memory $\mathrm{CD8}^{+} \mathrm{T}$ cells that contributes to the killing of intracellular $M$. tuberculosis is depleted in vivo by infliximab in patients with RA, and that these cells are susceptible to complement-mediated lysis in the presence of infliximab in vitro. The study provides insight into host defense mechanisms that act to control TB infection and how they are affected during anti-TNF immunotherapy for autoimmune disease.
\end{abstract}

Conflict of interest: The authors have declared that no conflict of interest exists.

Nonstandard abbreviations used: CDC, complement-dependent cytotoxicity; TB, tuberculosis; $\mathrm{T}_{\mathrm{EMRA}}$, $\mathrm{CD} 4 \mathrm{RA}^{+}$effector memory $\mathrm{T}$ (cell).

Citation for this article: J. Clin. Invest. 119:1079-1082 (2009). doi:10.1172/JCI39143.
TNF is a pleiotropic cytokine that plays a critical but incompletely understood role in immunity to Mycobacterium tuberculosis and other intracellular bacterial and fungal pathogens. TNF is not only essential for immune control of tuberculosis (TB), it has also been implicated in the immu- nopathology of the disease (1). While considerable knowledge of the actions of TNF in immunity to M. tuberculosis has been gained from studies in animal models, we have also gained a deeper understanding of TNF's contributions to the control of TB in humans through the use of TNF-neutralizing drugs for certain chronic inflammatory diseases. While these agents are highly efficacious for the treatment of RA, ankylosing spondylitis, psoriatic arthritis, and Crohn disease, they also promote reactivation (and possibly acquisition) of intracellular pathogens, including $M$. tuberculosis, resulting in potentially life-threatening infections. Since the early reports of $\mathrm{TB}$ in patients treated with infliximab, an anti-TNF monoclonal antibody, described abnormalities in granulomas (2), much of the work to elucidate the mechanisms of TB reactivation has focused on the roles of TNF in the formation and maintenance of these structures. Granulomas are thought to contribute to control of intramacro- 